\title{
Thiotepa-busulfan-fludarabine compared to busulfan-fludarabine for sibling and unrelated donor transplant in acute myeloid leukemia in first remission
}

\author{
Francesco Saraceni ${ }^{1}$, Myriam Labopin ${ }^{2}$, Rose-Marie Hamladji ${ }^{3}$, Ghulam Mufti ${ }^{4}$, Gerard \\ Socié $^{5}$, Avichai Shimoni ${ }^{6}$ Jeremy Delage7, Eric Deconinck ${ }^{8}$, Patrice Chevallier ${ }^{9}$, \\ Didier Blaise ${ }^{10}$, Jaime Sanz ${ }^{11}$, Anne Huynh ${ }^{12}$, Edouard Forcade ${ }^{13}$, Bipin N. Savani ${ }^{14}$, \\ Mohamad Mohty ${ }^{15}$, Arnon Nagler ${ }^{2,6}$ and Acute Leukemia Working Party of the \\ European society for Blood and Marrow Transplantation (EBMT) \\ ${ }^{1}$ Hematology and Stem Cell Transplant, Ravenna Hospital, Ravenna, Italy \\ ${ }^{2}$ EBMT Paris Study Office, Saint Antoine Hospital, Paris, France \\ ${ }^{3}$ Centre Pierre et Marie Curie, Service Hématologie Greffe de Moëlle, Alger, Algeria \\ ${ }^{4}$ GKT School of Medicine, Department of Haematological Medicine, London, United Kingdom \\ ${ }^{5}$ Hopital St. Louis, Department of Hematology-BMT, Paris, France \\ ${ }^{6}$ Chaim Sheba Medical Center, Department of Bone Marrow Transplantation, Tel-Hashomer, Israel \\ ${ }^{7} \mathrm{CHU}$ Lapeyronie, Département d'Hématologie Clinique, Montpellier, France \\ ${ }^{8}$ Hopital Jean Minjoz, Service d'Hématologie, Besançon, France \\ ${ }^{9} \mathrm{CHU}$ Nantes, Department D'Hématologie, Nantes, France \\ ${ }^{10}$ Programme de Transplantation and Therapie Cellulaire, Centre de Recherche en Cancérologie de Marseille, Institut Paoli \\ Calmettes, Marseille, France \\ ${ }^{11}$ Hospital Universitario La Fe, Servicio de Hematologia, Valencia, Spain \\ ${ }^{12}$ Institut Universitaire du Cancer Toulouse, Oncopole, I.U.C.T-O, Toulouse, France \\ ${ }^{13} \mathrm{CHU}$ Bordeaux, Hôpital Haut-Leveque, Pessac, France \\ ${ }^{14}$ Vanderbilt University Medical Center, Nashville, TN, USA \\ ${ }^{15}$ Department of Haematology, Saint Antoine Hospital, Paris, France \\ Correspondence to: Francesco Saraceni, email: francesco.saraceni@libero.it \\ Keywords: acute myeloid leukemia (AML); allogeneic transplantation; thiotepa-busulfan-fludarabine (TBF); busulfan-fludarabine \\ (BF); myeloablative conditioning (MAC) \\ Received: October 17, $2017 \quad$ Accepted: November 20, $2017 \quad$ Published: December 15, 2017 \\ Copyright: Saraceni et al. This is an open-access article distributed under the terms of the Creative Commons Attribution License 3.0 \\ ( $C$ C BY 3.0), which permits unrestricted use, distribution, and reproduction in any medium, provided the original author and source \\ are credited.
}

\section{ABSTRACT}

Background: A preparatory regimen consisting of thiotepa-busulfan-fludarabine (TBF) has been associated with reduced relapse in patients with haematological malignancies after haploidentical and cord blood transplants; however, few data exist regarding TBF conditioning in sibling (MSD) and unrelated donor (URD) transplants for AML.

Results: Among patients receiving a myeloablative (MAC) regimen, TBF-MAC was associated with significantly lower relapse (HR $0.47, p=0.005$ ) however higher non-relapse mortality (NRM, HR $2.69, p<10^{-4}$ ) as compared to BF. This led to similar leukemia-free (LFS) and overall survival (OS) between the two regimens (LFS: $p=0.6$; OS: $p=0.27$ ). When we selected TBF-MAC patients receiving busulfan $9.6 \mathrm{mg} / \mathrm{kg}$, NRM resulted still higher but no more significantly different as compared to BF-MAC with busulfan $12.8 \mathrm{mg} / \mathrm{kg}$ ( HR 1.53, $p=0.12$ ); despite the lower busulfan dose, 
relapse remained inferior with TBF-MAC (HR 0.45, $p=0.01$ ), however no difference in survival could be demonstrated (LFS: $p=0.31 ;$ OS: 0.82 ). Among patients receiving a reduced-intensity (RIC) regimen, similar outcome was observed with TBF-RIC and BF-RIC (LFS: $p=0.77$; OS: $p=0.88$ ).

Conclusions: TBF-MAC as conditioning regimen for transplant from MSD and URD in AML patients in first remission provided stronger anti-leukemic activity but higher NRM as compared to BF-MAC, thus leading to similar survival. TBF-MAC with busulfan $9.6 \mathrm{mg} / \mathrm{kg}$ was associated with low relapse and acceptable NRM, however again with no survival benefit. TBF-RIC and BF-RIC resulted in comparable outcome.

Methods: We conducted a registry-based study comparing outcomes of patients with AML in first remission undergoing transplant from MSD or URD prepared with either TBF $(n=212)$ or BF $(n=2698)$ conditioning.

\section{INTRODUCTION}

In recent years, the paradigm of conditioning regimens for allogeneic stem cell transplant (allo-SCT) in AML has shifted from heavy myeloablation to reduced intensity protocols, with the aim of minimizing regimen related toxicity while exploiting the graft-versus-leukemia effect [1]. An important step towards this goal has been made with the replacement of cyclophosphamide with fludarabine [2]. A preparatory regimen consisting of busulfan and fludarabine (BF) was initially described in the early 2000s; [3] subsequently different studies were carried out to challenge $\mathrm{BF}$ with standard $\mathrm{BuCy}$, reporting conflicting results on the relative merits of the two regimens in terms of non-relapse mortality (NRM), relapse and survival [4-7]. More recently, different prospective randomized trials have been conducted in an attempt to answer the question whether BF could be a valid substitution for standard $\mathrm{BuCy}$, reporting inconsistent results [8-11]. A recent meta-analysis of 15 studies [12] concluded that the $\mathrm{BF}$ conditioning regimen is associated with inferior toxicity and lower 100-days NRM with no increase in relapse risk as compared to $\mathrm{BuCy}$; however, no survival benefit could be demonstrated. In fact, although the substitution of cyclophosphamide with fludarabine results in decreased toxicity, leukemia relapse still represents the main cause of transplant failure, accounting for about $30 \%$ at 2 years when transplant is performed in remission.

Thiotepa is an alkylating compound with immunosuppressive properties which holds favourable characteristics as the capability of penetrating the bloodbrain barrier combined with a reduced non-haematologic toxicity, which led to a widespread use of this compound within transplant preparative regimens [13-15]. A conditioning protocol consisting of thiotepa, busulfan and fludarabine (TBF), initially designed for cord blood transplant, $[16,17]$ has recently been associated with low relapse rates and satisfactory outcome in haploidentical transplantation (haplo-SCT) [18-22] and in single center reports of allo-SCT from MSD and URD-SCT for different haematological malignancies [23, 24]. However, no data are available about outcome of AML patients undergoing MSD or URD-SCT prepared with this protocol, and no comparison of this novel regimen with standard regimens has been published yet. We therefore took advantage of the EBMT dataset, and retrospectively compared outcome following TBF versus $\mathrm{BF}$ as conditioning regimens before allo-SCT from MSD or URD-SCT in a large homogeneous population of AML patients undergoing transplant in first complete remission (CR1).

\section{RESULTS}

\section{Patient characteristics}

Patients from 204 transplant centers were included. The total number of patients who received either TBF or BF in CR1 between January 2007 and June 2015 and reported to the EBMT was 4995 (265 TBF and $4730 \mathrm{BF}$ ); two thousand and eighty-five patients were excluded from the analysis as they have received a busulfan dose $<6.4 \mathrm{mg} / \mathrm{kg}$, thus leading to a final number of 2910 patients; among them, 212 received TBF and $2698 \mathrm{BF}$, respectively. One thousand six hundred and six patients received a MAC and 1304 a RIC regimen. One thousand six hundred and fifty-three (57\%) patients were transplanted from MSD, 987 (34\%) from 10/10 URD and 9\% (270) from 9/10 URD, respectively. Data about cytogenetic risk were available for $55 \%$ of patients, while information on molecular genetics and minimal residual disease (MRD) status was missing for most patients and was therefore not included in the analysis. The characteristics of patients are summarized in Table 1.

\section{Myeloablative conditioning: TBF-MAC versus BF-MAC}

\section{Patient, disease and transplant characteristics}

One-hundred and forty-seven patients received TBF-MAC, while 1459 patients received the BF-MAC regimen. TBF-MAC group included significantly younger patients (median age 45 vs 50 years, $p<10^{-3}$ ) transplanted more recently (median year of transplant 2014 vs 2013, $p<10^{-3}$ ) as compared to for BF-MAC, respectively. Patients conditioned with TBF-MAC were more likely to 


\begin{tabular}{|c|c|c|c|c|c|c|c|}
\hline & & \multicolumn{4}{|c|}{ Type of transplant } & \multicolumn{2}{|c|}{$P$} \\
\hline & & \multicolumn{2}{|c|}{ MAC } & \multicolumn{2}{|c|}{ RIC } & \multirow[t]{2}{*}{ MAC } & \multirow[t]{2}{*}{ RIC } \\
\hline & & TBF-MAC & BF-MAC & TBF-RIC & BF-RIC & & \\
\hline \multicolumn{2}{|l|}{ Number (total: 2910) } & 147 & 1459 & 65 & 1239 & & \\
\hline \multicolumn{2}{|c|}{ Follow up, months (range) } & $15(1-75)$ & $19(1-111)$ & $19(1-111)$ & $15(1-75)$ & & \\
\hline \multicolumn{2}{|c|}{ Age at transplant, years (range; IQR) } & $45(19-66 ; 34-52)$ & $50(18-74 ; 39-57)$ & $60(27-72 ; 56-63)$ & $60(19-75 ; 54-64)$ & $<10^{-3}$ & 0.79 \\
\hline \multicolumn{2}{|c|}{ Median year of transplant (range) } & $2014(2008-2015)$ & $2013(2007-2015)$ & $2014(2008-2015)$ & $2012(2007-2015)$ & $<10^{-3}$ & $<10^{-3}$ \\
\hline \multirow[t]{5}{*}{$\begin{array}{l}\text { Cytogenetic risk, } \\
n(\%)\end{array}$} & Favorable & $9(16 \%)$ & $65(11 \%)$ & $1(3 \%)$ & $53(6 \%)$ & 0.285 & 0.093 \\
\hline & Intermediate-I & $7(13 \%)$ & $122(20 \%)$ & $6(21 \%)$ & $250(28 \%)$ & & \\
\hline & Intermediate-II & $16(29 \%)$ & $125(20 \%)$ & $3(10 \%)$ & $160(18 \%)$ & & \\
\hline & Adverse & $6(11 \%)$ & $88(14 \%)$ & $2(7 \%)$ & $128(14 \%)$ & & \\
\hline & missing & 91 & 848 & 36 & 348 & & \\
\hline \multirow[t]{2}{*}{ Diagnosis, $n$ (\%) } & De novo AML & $129(88 \%)$ & $1248(86 \%)$ & $48(74 \%)$ & $939(76 \%)$ & 0.464 & 0.722 \\
\hline & Secondary AML & $18(12 \%)$ & $211(14 \%)$ & $17(26 \%)$ & $300(24 \%)$ & & \\
\hline \multirow[t]{4}{*}{ Karnofsky PS, $n$ (\%) } & $<80 \%$ & $2(1 \%)$ & $19(1 \%)$ & $1(2 \%)$ & $59(5 . \%)$ & 0.985 & 0.227 \\
\hline & $\geq 80 \%$ & $145(99 \%)$ & $1358(99 \%)$ & $59(98 \%)$ & $1088(94 \%)$ & & \\
\hline & $<90 \%$ & $17(12 \%)$ & $207(15 \%)$ & $12(20 \%)$ & $291(25 \%)$ & 0.259 & 0.342 \\
\hline & $\geq 90 \%$ & $130(88 \%)$ & $1170(85 \%)$ & $48(80 \%)$ & $852(74 \%)$ & & \\
\hline \multirow[t]{3}{*}{ Donor, $n(\%)$} & MSD & $75(51 \%)$ & $944(65 \%)$ & $39(60 \%)$ & $595(48 \%)$ & 0.001 & 0.113 \\
\hline & 10/10 URD & $50(34 \%)$ & $396(27 \%)$ & $23(35 \%)$ & $518(42 \%)$ & & \\
\hline & 9/10 URD & $22(15 \%)$ & $119(8 \%)$ & $3(5 \%)$ & $126(10 \%)$ & & \\
\hline \multirow[t]{2}{*}{$\begin{array}{l}\text { Stem cell source, } \\
n(\%)\end{array}$} & $\mathrm{BM}$ & $50(34 \%)$ & $247(17 \%)$ & $11(17 \%)$ & $62(5 \%)$ & $<10^{-3}$ & $<10^{-3}$ \\
\hline & PBSC & $97(66 \%)$ & $1212(83 \%)$ & $54(83 \%)$ & $1177(95 \%)$ & & \\
\hline \multirow[t]{2}{*}{ Patient gender, $n$ (\%) } & male & $74(50 \%)$ & $787(54 \%)$ & $38(59 \%)$ & $657(53 \%)$ & 0.38 & 0.327 \\
\hline & female & $73(50 \%)$ & $667(46 \%)$ & $26(41 \%)$ & $580(47 \%)$ & & \\
\hline \multirow[t]{3}{*}{ Donor gender, $\boldsymbol{n}$ (\%) } & Male & $102(69 \%)$ & $858(59 \%)$ & $35(55 \%)$ & $772(63 \%)$ & 0.018 & 0.179 \\
\hline & Female & $45(31 \%)$ & $587(41 \%)$ & $29(45 \%)$ & $453(37 \%)$ & & \\
\hline & Missing & 0 & 14 & 1 & 14 & & \\
\hline \multirow{3}{*}{$\begin{array}{l}\text { Donor/recipient } \\
\text { gender matching, } \\
n(\%)\end{array}$} & No $F$ to $M$ & $122(83 \%)$ & $1126(78 \%)$ & $48(75 \%)$ & $1010(83 \%)$ & 0.176 & 0.122 \\
\hline & $\mathrm{F}$ to $\mathrm{M}$ & $25(17 \%)$ & $314(22 \%)$ & $16(25 \%)$ & $213(17 \%)$ & & \\
\hline & Missing & 0 & 19 & 1 & 16 & & \\
\hline \multirow[t]{3}{*}{$\begin{array}{l}\text { Patient CMV } \\
\text { serology, } n(\%)\end{array}$} & negative & $31(22 \%)$ & $324(23 \%)$ & $8(13 \%)$ & $417(34 \%)$ & 0.806 & $<10^{-4}$ \\
\hline & positive & $112(78 \%)$ & $1111(77 \%)$ & $55(87 \%)$ & $808(66 \%)$ & & \\
\hline & Missing & 4 & 24 & 2 & 14 & & \\
\hline \multirow[t]{3}{*}{$\begin{array}{l}\text { Donor CMV serology, } \\
n(\%)\end{array}$} & negative & $56(39 \%)$ & $485(34 \%)$ & $18(29 \%)$ & $580(47 \%)$ & 0.229 & 0.003 \\
\hline & positive & $88(61 \%)$ & $946(66 \%)$ & $45(71 \%)$ & $643(53 \%)$ & & \\
\hline & Missing & 3 & 28 & 2 & 16 & & \\
\hline \multirow[t]{5}{*}{$\begin{array}{l}\text { CMV donor/recipient } \\
\text { matching }\end{array}$} & $\mathrm{D}-/ \mathrm{R}-$ & $18(13 \%)$ & $215(15 \%)$ & $3(5 \%)$ & $300(25 \%)$ & 0.297 & \\
\hline & $\mathrm{D}+/ \mathrm{R}_{-}$ & $12(9 \%)$ & $107(8 \%)$ & $5(8 \%)$ & $117(10 \%)$ & & \\
\hline & $\mathrm{D}-/ \mathrm{R}+$ & $35(25 \%)$ & $264(19 \%)$ & $15(24 \%)$ & $278(23 \%)$ & & \\
\hline & $\mathrm{D}+/ \mathrm{R}+$ & $76(54 \%)$ & $830(59 \%)$ & $39(63 \%)$ & $519(43 \%)$ & & \\
\hline & Missing & 6 & 43 & 3 & 25 & & \\
\hline in vivo TCD, $n(\%)$ & No & $67(46 \%)$ & $646(45 \%)$ & $39(60 \%)$ & $213(17 \%)$ & 0.718 & $<10^{-4}$ \\
\hline
\end{tabular}




\begin{tabular}{|c|c|c|c|c|c|c|c|}
\hline & Yes & $78(54 \%)$ & $801(55 \%)$ & $26(40 \%)$ & $1024(83 \%)$ & & \\
\hline & Missing & 2 & 12 & 0 & 2 & & \\
\hline \multirow[t]{6}{*}{ GVHD prophylaxis } & $\mathrm{CSA}+\mathrm{ATG}$ & $9(6.3 \%)$ & $175(12.4 \%)$ & $6(9.2 \%)$ & $391(32.1 \%)$ & 0.002 & $<0.001$ \\
\hline & $\mathrm{CSA}+\mathrm{MTX}$ & $50(34.5 \%)$ & $527(37.2 \%)$ & $21(32.3 \%)$ & $139(11.4 \%)$ & & \\
\hline & $\begin{array}{l}\text { CSA + MTX + } \\
\text { ATG }\end{array}$ & $62(43.1 \%)$ & $386(27.2 \%)$ & $16(24.6 \%)$ & $208(17.1 \%)$ & & \\
\hline & $\mathrm{CSA}+\mathrm{MMF}$ & $6(4.2 \%)$ & $60(4.2 \%)$ & $3(4.6 \%)$ & $34(2.8 \%)$ & & \\
\hline & $\begin{array}{l}\mathrm{CSA}+\mathrm{MMF}+ \\
\mathrm{ATG}\end{array}$ & $6(4.2 \%)$ & $110(7.8 \%)$ & $3(4.6 \%)$ & $319(26.2 \%)$ & & \\
\hline & other & $11(7.6 \%)$ & $159(11.2 \%)$ & $16(24.6 \%)$ & $127(10.4 \%)$ & & \\
\hline \multirow[t]{2}{*}{ Busulfan dose, $n(\%)$} & $9.6 \mathrm{mg} / \mathrm{Kg}$ & $111(76 \%)$ & $339(23 \%)$ & $111(76 \%)$ & $339(23 \%)$ & $<10^{-3}$ & $<10^{-3}$ \\
\hline & $12.8 \mathrm{mg} / \mathrm{Kg}$ & $36(24 \%)$ & $1120(77 \%)$ & $36(24 \%)$ & $1120(77 \%)$ & & \\
\hline
\end{tabular}

Data are median (IQR), median (range), median (range; IQR), $n(\%)$, or $\mathrm{n} / \mathrm{N}(\%)$. Some percentages do not add up to $100 \%$ because of rounding. Abbreviations: BF, busulfan-fludarabine; BM, Bone marrow; CMV, Cytomegalovirus; MAC, myeloablative conditioning; MSD, matched sibling donor; URD, unrelated donor; PBSCs, peripheral blood stem cells; RIC, reduced-intensity conditioning; TBF, thiotepa-busulfan-fludarabine; TCD, T-cell depletion.

have received a URD transplant ( $49 \%$ vs $35 \%, p=0.001)$, $\mathrm{BM}$ as stem cell source $\left(34 \%\right.$ vs $\left.17 \%, p<10^{-3}\right)$, and the donor most likely to be male ( $69 \%$ vs $59 \%, p=0.018)$, as compared to BF-MAC. Cytogenetic risk, proportion of patients with secondary AML, Karnofsky performance score, proportion of patients who received in-vivo T-cell depletion, kind of donor and patient CMV serology did not differ between the groups. Among the TBF-MAC cohort, 111 patients $(76 \%)$ received busulfan $9.6 \mathrm{mg} / \mathrm{kg}$, while 36 (24\%) received $12.8 \mathrm{mg} / \mathrm{kg}$. Within the BF-MAC cohort, 339 patients (23\%) received busulfan $9.6 \mathrm{mg} / \mathrm{kg}$ and 1120 (77\%) $12.8 \mathrm{mg} / \mathrm{kg}$.

\section{Engraftment, NRM and GVHD}

Engraftment rate was $98 \%$ following both regimens $(p=0.88)$. The median time to neutrophil engraftment was $18(10-47)$ days and 15 (5-45) days for TBF-MAC and BF-MAC, respectively $\left(p<10^{-5}\right)$. The 2-year non-relapse mortality rate in the overall population was significantly higher after TBF-MAC compared to BF-MAC in both univariate $(27 \pm 8 \%$ vs $16 \pm 2 \%, p=0.006)$ and multivariate analysis (HR 2.7, $p<10^{-4}$ ). When analyzing separately NRM within and after day 100, NRM rate following TBF-MAC was significantly higher as compared to BFMAC within 100 days after transplant ( $8 \pm 4 \%$ vs $5 \pm 1 \%$, $p=0.008$ ), while a trend for higher NRM was observed for the time period between 100 days and 2 years after transplant (19 $\pm 8 \%$ vs $12 \pm 2 \%$ for TBF-MAC and BFMAC respectively, $p=0.08)$. Karnofsky performance score at transplant ( $\geq 90 \%$ vs $<90 \%$ ) did not affect risk of NRM. Independent predictive factors for higher NRM risk were older age, higher busulfan dose, URD transplant compared to MSD and PBSC compared to BM graft.

Main causes of NRM were GVHD and infectious complications (Table 2). When analyzing separately the incidence of infectious-related and GVHD-related deaths no difference was observed between the two study cohorts.

Veno-occlusive disease (VOD) accounted for $2(5 \%)$ of 43 lethal complications in the TBF and $8(2 \%)$ of 478 deaths in the BF group, respectively.

The incidence of grade II-IV and III-IV acute graftversus-host disease (aGVHD) was similar between the groups, being $25 \pm 7 \%$ and $10 \pm 5 \%$ in the TBF-MAC and $24 \pm 2 \%$ and $7 \pm 1 \%$ in BF-MAC groups, $(p=0.83$ and $p=0.22$, respectively). The 2 -year cumulative incidence of cGVHD of any grade was similar following TBF-MAC $(35 \pm 9 \%)$ compared to BF-MAC $(40 \pm 3 \%, p=0.5)$. Similarly, no difference was observed as for the incidence of severe cGVHD ( $17 \%$ in both groups, $p=0.78)$. Multivariate analysis confirmed those results (Table 3).

\section{Transplant outcome}

The 2-year relapse incidence was significantly lower in TBF-MAC group (14 $\pm 6 \%$ ) compared to BF-MAC group (27 $\pm 2 \%, p=0.002)$ (Figure 1). This result was strongly confirmed in multivariate analysis ( $\mathrm{HR} 0.5, p=$ 0.005). Secondary AML was the only additional factor associated with higher relapse risk in multivariate analysis (Table 3).

The reduced relapse following TBF-MAC was particularly evident in the early post transplant period; in fact, relapse incidence before day 100 was $2 \%(95 \% \mathrm{CI}$ : $1-6)$ for TBF-MAC vs $9 \%$ (95\% CI: 7-10) for BF-MAC $(p=0.007)$, while no significant difference in relapse incidence was observed after day 100 (13\% (95\% CI: $7-20)$ vs $17 \%$ (95\% CI: $14-19)$ for TBF-MAC and BFMAC respectively, $p=0.4)$.

Leukemia-free survival (LFS) at 2 years was not statistically different between TBF-MAC and BF-MAC groups, being $59 \pm 10 \%$ in TBF-MAC and $57 \pm 3 \%$ in BF-MAC, respectively $(p=0.5)$ (Figure 2). Multivariate 
Table 2A: Causes of death

\begin{tabular}{|c|c|c|c|c|}
\hline & \multicolumn{2}{|c|}{ MAC } & \multicolumn{2}{|c|}{ RIC } \\
\hline & TBF & BF & TBF & BF \\
\hline Total & 43 & 501 & 18 & 468 \\
\hline GVHD & $13(32 \%)$ & $100(21 \%)$ & $4(24 \%)$ & $100(22 \%)$ \\
\hline Infection & $10(24 \%)$ & $89(19 \%)$ & $3(18 \%)$ & $78(17 \%)$ \\
\hline Original disease & $12(29 \%)$ & $247(52 \%)$ & $8(47 \%)$ & $234(52 \%)$ \\
\hline VOD & $2(5 \%)$ & $8(2 \%)$ & 0 & 0 \\
\hline Failure/Rejection & 0 & $2(1 \%)$ & 0 & $2(1 \%)$ \\
\hline Hemorrhage & $1(2 \%)$ & $1(1 \%)$ & 0 & $3(1 \%)$ \\
\hline Cardiac toxicity & 0 & $1(1 \%)$ & $1(6 \%)$ & $1(1 \%)$ \\
\hline $\begin{array}{l}\text { Interstitial } \\
\text { Pneumonitis }\end{array}$ & 0 & $6(2 \%)$ & $1(6 \%)$ & $3(1 \%)$ \\
\hline Second malignancy & $1(2 \%)$ & $3(1 \%)$ & 0 & $6(2 \%)$ \\
\hline $\begin{array}{l}\text { Other transplantation } \\
\text { related }\end{array}$ & $2(5 \%)$ & $21(4 \%)$ & 0 & $19(4 \%)$ \\
\hline Missing & 2 & 23 & 1 & 22 \\
\hline
\end{tabular}

Table 2B: Causes of death by time period after transplant

\begin{tabular}{|c|c|c|c|c|c|}
\hline & & \multicolumn{2}{|c|}{ MAC } & \multicolumn{2}{|c|}{ RIC } \\
\hline & & TBF & BF & TBF & BF \\
\hline \multirow[t]{12}{*}{ Before day 100} & Total & 14 & 184 & 8 & 157 \\
\hline & Infection & $4(29 \%)$ & $39(21 \%)$ & $3(38 \%)$ & $20(13 \%)$ \\
\hline & GVHD & $4(29 \%)$ & $31(17 \%)$ & 0 & $24(15 \%)$ \\
\hline & Original disease & $2(14 \%)$ & $89(49 \%)$ & $3(38 \%)$ & $100(65 \%)$ \\
\hline & Cardiac toxicity & 0 & 0 & $1(13 \%)$ & $1(1 \%)$ \\
\hline & Haemorhage & $1(7 \%)$ & $1(1 \%)$ & 0 & $1(1 \%)$ \\
\hline & Failure/Rejection & 0 & $2(1.1 \%)$ & 0 & 0 \\
\hline & VOD & $2(14 \%)$ & $8(4 \%)$ & 0 & 0 \\
\hline & $\begin{array}{c}\text { Interstitial } \\
\text { Pneumonitis }\end{array}$ & 0 & 2 & $1(13 \%)$ & $1(1 \%)$ \\
\hline & $\begin{array}{c}\text { Second } \\
\text { malignancy }\end{array}$ & 0 & 0 & 0 & 0 \\
\hline & $\begin{array}{c}\text { Other } \\
\text { transplantation } \\
\text { related }\end{array}$ & $1(7 \%)$ & $10(5 \%)$ & 0 & $8(5 \%)$ \\
\hline & Missing & 0 & 2 & 0 & 2 \\
\hline \multirow[t]{8}{*}{$\begin{array}{l}\text { From day } 100 \text { to } \\
2 \text { years }\end{array}$} & Total & 25 & 274 & 8 & 259 \\
\hline & Original disease & $9(38 \%)$ & $141(54 \%)$ & $4(57 \%)$ & $112(46 \%)$ \\
\hline & GVHD & $8(33 \%)$ & $59(23 \%)$ & $3(43 \%)$ & $71(29 \%)$ \\
\hline & Infection & $5(21 \%)$ & $44(17 \%)$ & 0 & $44(18 \%)$ \\
\hline & Haemorhage & 0 & 0 & 0 & $2(1 \%)$ \\
\hline & Failure/Rejection & 0 & 0 & 0 & $2(1 \%)$ \\
\hline & VOD & 0 & 0 & 0 & 0 \\
\hline & Cardiac toxicity & 0 & $1(1 \%)$ & 0 & 0 \\
\hline
\end{tabular}




\begin{tabular}{ccccc}
$\begin{array}{c}\text { Interstitial } \\
\text { Pneumonitis } \\
\begin{array}{c}\text { Second } \\
\text { malignancy }\end{array}\end{array}$ & 0 & $4(2 \%)$ & 0 & $2(1 \%)$ \\
$\begin{array}{c}\text { Other } \\
\text { transplantation } \\
\text { related } \\
\text { Missing }\end{array}$ & $1(4 \%)$ & $1(1 \%)$ & 0 & $3(1 \%)$ \\
& 1 & $10(4 \%)$ & 0 & $8(3 \%)$ \\
\hline
\end{tabular}

Abbreviations: BF, busulfan-fludarabine; GVHD, graft-versus-host disease; MAC, myeloablative conditioning; RIC, reduced-intensity conditioning; TBF, thiotepa-busulfan-fludarabine; VOD, veno-occlusive disease.

Table 3: Multivariate analysis of transplantation outcome

\begin{tabular}{|c|c|c|c|c|c|c|c|}
\hline & \multirow[b]{2}{*}{ Outcome } & \multicolumn{3}{|c|}{ MAC } & \multicolumn{3}{|c|}{ RIC } \\
\hline & & HR & 95\% CI & $P$ & HR & $95 \% \mathrm{CI}$ & $P$ \\
\hline \multirow[t]{9}{*}{ Relapse } & TBF vs BF & 0.47 & $0.27-0.80$ & 0.005 & 0.98 & $0.56-1.7$ & 0.9 \\
\hline & Patient age per 10 years & 1.09 & $0.99-1.20$ & 0.08 & 1.05 & $0.93-1.19$ & 0.4 \\
\hline & Secondary AML & 1.45 & $1.11-1.90$ & 0.007 & 1.36 & $1.07-1.72$ & 0.011 \\
\hline & URD vs MSD & 0.97 & $0.75-1.25$ & 0.79 & 0.77 & $0.61-0.97$ & 0.02 \\
\hline & $\begin{array}{l}\text { Female donor to male } \\
\text { recipient }\end{array}$ & 0.79 & $0.60-1.03$ & 0.078 & 1.04 & $0.78-1.37$ & 0.8 \\
\hline & In-vivo TCD & 0.96 & $0.74-1.23$ & 0.7 & 0.99 & $0.72-1.37$ & 0.97 \\
\hline & Dose $\mathrm{Bu} 12.8$ vs $9.6 \mathrm{mg} / \mathrm{kg}$ & 0.89 & $0.69-1.15$ & 0.37 & & & \\
\hline & PBSC vs BM & 1.01 & $0.76-1.3$ & 0.95 & 0.84 & $0.56-1.28$ & 0.42 \\
\hline & centre (frailty) & & & 0.29 & & & 0.18 \\
\hline \multirow[t]{9}{*}{ NRM } & TBF vs BF & 2.69 & $1.61-4.39$ & $<10^{-4}$ & 0.79 & $0.39-1.58$ & 0.5 \\
\hline & Patient age per 10 years & 1.55 & $1.35-1.77$ & $<10^{-4}$ & 1.27 & $1.06-1.52$ & 0.01 \\
\hline & Secondary AML & 0.89 & $0.61-1.3$ & 0.55 & 1.39 & $1.01-1.9$ & 0.04 \\
\hline & URD vs MSD & 1.51 & $1.07-2.14$ & 0.019 & 1.8 & $1.32-2.57$ & 0.001 \\
\hline & $\begin{array}{l}\text { Female donor to male } \\
\text { recipient }\end{array}$ & 1.12 & $0.82-1.53$ & 0.47 & 1.49 & $1.05-2.12$ & 0.025 \\
\hline & In-vivo $\mathrm{TCD}$ & 0.86 & $0.61-1.21$ & 0.38 & 0.72 & $0.47-1.09$ & 0.12 \\
\hline & Dose $\mathrm{Bu} 12.8$ vs 9.6 mg/kg & 1.67 & $1.15-2.43$ & 0.007 & & & \\
\hline & PBSC vs BM & 1.73 & $1.14-2.65$ & 0.01 & 0.79 & $0.45-1.40$ & 0.42 \\
\hline & centre (frailty) & & & 0.001 & & & 0.71 \\
\hline \multirow[t]{9}{*}{ LFS } & TBF vs BF & 1.09 & $0.77-1.54$ & 0.6 & 0.94 & $0.61-1.44$ & 0.77 \\
\hline & Patient age per 10 years & 1.22 & $1.13-1.33$ & $<10^{-4}$ & 1.13 & $1.02-1.25$ & 0.02 \\
\hline & Secondary AML & 1.22 & $0.98-1.52$ & 0.07 & 1.39 & $1.15-1.67$ & 0.001 \\
\hline & URD vs MSD & 1.13 & $0.92-1.40$ & 0.24 & 1.02 & $0.85-1.23$ & 0.85 \\
\hline & $\begin{array}{l}\text { Female donor to male } \\
\text { recipient }\end{array}$ & 0.91 & $0.74-1.11$ & 0.36 & 1.17 & $0.94-1.45$ & 0.16 \\
\hline & In-vivo $\mathrm{TCD}$ & 0.9 & $0.73-1.11$ & 0.32 & 0.89 & $0.69-1.14$ & 0.35 \\
\hline & Dose $\mathrm{Bu} 12.8$ vs $9.6 \mathrm{mg} / \mathrm{kg}$ & 1.15 & $0.93-1.42$ & 0.21 & & & \\
\hline & PBSC vs BM & 1.25 & $0.99-1.58$ & 0.06 & 0.8 & $0.58-1.13$ & 0.22 \\
\hline & centre (frailty) & & & 0.13 & & & 0.88 \\
\hline \multirow[t]{4}{*}{ OS } & TBF vs BF & 1.23 & $0.85-1.79$ & 0.27 & 0.77 & $0.46-1.27$ & 0.3 \\
\hline & Patient age per 10 years & 1.28 & $1.18-1.4$ & $<10^{-4}$ & 1.17 & $1.05-1.31$ & 0.006 \\
\hline & Secondary AML & 1.15 & $0.91-1.46$ & 0.23 & 1.44 & $1.18-1.76$ & 0.001 \\
\hline & URD vs MSD & 1.01 & $0.88-1.37$ & 0.4 & 1.25 & $1.02-1.53$ & 0.03 \\
\hline
\end{tabular}




\begin{tabular}{|c|c|c|c|c|c|c|c|}
\hline & $\begin{array}{l}\text { Female donor to male } \\
\text { recipient }\end{array}$ & 0.92 & $0.74-1.14$ & 0.45 & 1.3 & $1.03-1.63$ & 0.03 \\
\hline & In-vivo TCD & 0.92 & $0.73-1.16$ & 0.48 & 0.79 & $0.602-1.036$ & 0.09 \\
\hline & Dose $\mathrm{Bu} 12.8$ vs $9.6 \mathrm{mg} / \mathrm{kg}$ & 1.09 & $0.87-1.38$ & 0.45 & & & \\
\hline & PBSC vs BM & 1.34 & $1.04-1.74$ & 0.026 & 0.83 & $0.58-1.20$ & 0.32 \\
\hline & centre (frailty) & & & 0.057 & & & 0.89 \\
\hline \multirow[t]{9}{*}{ GRFS } & TBF vs BF & 1 & $0.72-1.40$ & 0.99 & 0.79 & $0.52-1.20$ & 0.27 \\
\hline & Patient age per 10 years & 1.17 & $1.09-1.26$ & $<10^{-4}$ & 1.07 & $0.97-1.17$ & 0.18 \\
\hline & Secondary AML & 1.11 & $0.91-1.37$ & 0.3 & 1.24 & $1.04-1.48$ & 0.02 \\
\hline & URD vs MSD & 1.06 & $0.88-1.29$ & 0.56 & 1.11 & $0.94-1.32$ & 0.23 \\
\hline & $\begin{array}{l}\text { Female donor to male } \\
\text { recipient }\end{array}$ & 1.16 & $0.97-1.37$ & 0.1 & 1.16 & $0.95-1.42$ & 0.14 \\
\hline & In-vivo TCD & 0.83 & $0.68-1.02$ & 0.07 & 0.72 & $0.57-0.90$ & 0.005 \\
\hline & Dose $\mathrm{Bu} 12.8$ vs 9.6 mg $/ \mathrm{kg}$ & 1.06 & $0.86-1.30$ & 0.6 & & & \\
\hline & PBSC vs BM & 1.26 & $1.00-1.57$ & 0.05 & 0.99 & $0.71-1.37$ & 0.95 \\
\hline & centre (frailty) & & & 0.005 & & & 0.19 \\
\hline \multirow[t]{9}{*}{ aGVHD III-IV } & TBF vs BF & 1.34 & $0.71-2.53$ & 0.37 & 0.69 & $0.23-2.04$ & 0.5 \\
\hline & Patient age per 10 years & 1.07 & $0.91-1.26$ & 0.44 & 0.99 & $0.79-1.26$ & 0.98 \\
\hline & Secondary AML & 0.99 & $0.57-1.7$ & 0.97 & 1.12 & $0.70-1.78$ & 0.64 \\
\hline & URD vs MSD & 1.08 & $0.68-1.71$ & 0.75 & 3.09 & $1.87-5.10$ & $<10^{-4}$ \\
\hline & $\begin{array}{l}\text { Female donor to male } \\
\text { recipient }\end{array}$ & 1.03 & $0.65-1.6$ & 0.89 & 1.46 & $0.87-2.44$ & 0.15 \\
\hline & In-vivo TCD & 0.89 & $0.57-1.37$ & 0.57 & 0.54 & $0.29-1.08$ & 0.05 \\
\hline & Dose $\mathrm{Bu} 12.8$ vs $9.6 \mathrm{mg} / \mathrm{kg}$ & 0.85 & $0.54-1.34$ & 0.49 & & & \\
\hline & PBSC vs BM & 1.89 & $1.05-3.42$ & 0.03 & 2.05 & $0.64-6.61$ & 0.23 \\
\hline & centre (frailty) & & & 0.75 & & & 0.04 \\
\hline \multirow[t]{9}{*}{$\begin{array}{l}\text { Severe } \\
\text { cGVHD }\end{array}$} & TBF vs BF & 1.13 & $0.6-2.12$ & 0.71 & 0.46 & $0.15-1.35$ & 0.16 \\
\hline & Patient age per 10 years & 1.13 & $0.99-1.30$ & 0.08 & 0.95 & $0.8-1.14$ & 0.6 \\
\hline & Secondary AML & 1.01 & $0.65-1.57$ & 0.98 & 0.94 & $0.62-1.42$ & 0.78 \\
\hline & URD vs MSD & 1.3 & $0.87-1.95$ & 0.20 & 1.18 & $0.82-1.71$ & 0.38 \\
\hline & $\begin{array}{l}\text { Female donor to male } \\
\text { recipient }\end{array}$ & 1.91 & $1.42-2.58$ & $<10^{-4}$ & 1.26 & $0.83-1.89$ & 0.27 \\
\hline & In-vivo TCD & 0.33 & $0.21-0.51$ & $<10^{-4}$ & 0.48 & $0.30-0.78$ & 0.003 \\
\hline & Dose $\mathrm{Bu} 12.8$ vs $9.6 \mathrm{mg} / \mathrm{kg}$ & 1.15 & $0.75-1.76$ & 0.52 & & & \\
\hline & PBSC vs BM & 1.54 & $0.98-2.42$ & 0.06 & 1.2 & $0.57-2.54$ & 0.63 \\
\hline & centre (frailty) & & & 0.002 & & & 0.02 \\
\hline
\end{tabular}

Abbreviations: aGVHD, acute graft-versus-host disease; BF, busulfan-fludarabine; BM, Bone marrow; Bu, busulfan; cGVHD, chronic graft-versus-host disease; GRFS, graft-versus-host-free, relapse-free survival; MAC, myeloablative conditioning; LFS, leukemia-free survival; MSD, matched sibling donor; NRM, non-relapse mortality OS, overall survival; URD, unrelated donor; PBSCs, peripheral blood stem cells; RIC, reduced-intensity conditioning; TBF, thiotepa-busulfanfludarabine; TCD, T-cell depletion.

analysis confirmed those results. The only predictive factor independently associated with LFS in multivariate analysis was age at transplant. Overall survival at 2 years also did not differ between the two regimens, being $62 \pm 10 \%$ and $61 \pm 3 \%$ in TBF-MAC and BF-MAC, respectively $(p=0.87)$. This result was confirmed by multivariate analysis. Factors which negatively affected survival in multivariate analysis were older age at transplant and PBSCs as stem cell source. The composite endpoint GVHD-free, relapse-free survival (GRFS) rate at 2 years did not differ between the 2 conditioning regimens being $52 \%$ in TBF-MAC and $41 \%$ in BF-MAC, respectively $(p=0.2)$. Importantly, the use of ATG was independently associated with significantly lower probability of 
severe cGVHD and with a trend for better GRFS. In the population of patients who relapsed after transplant, there was no difference in overall survival after relapse between the two study cohorts. In MAC group 2-year after relapse OS was $14 \%$ vs $10 \%$ in the TBF and BF groups, respectively ( $p=0.74)$. In RIC cohort OS was $17 \%$ vs $13 \%$ one year after relapse $(p=0.86)$.

\section{Subgroup analysis}

In multivariate analysis conducted in the global population, busulfan dose (12.8 vs $9.6 \mathrm{mg} / \mathrm{kg}$ ) emerged as independently associated with NRM, while had no impact on relapse risk. Given this findings, and according to the regimens schedules as previously published, $[10$,
NRM

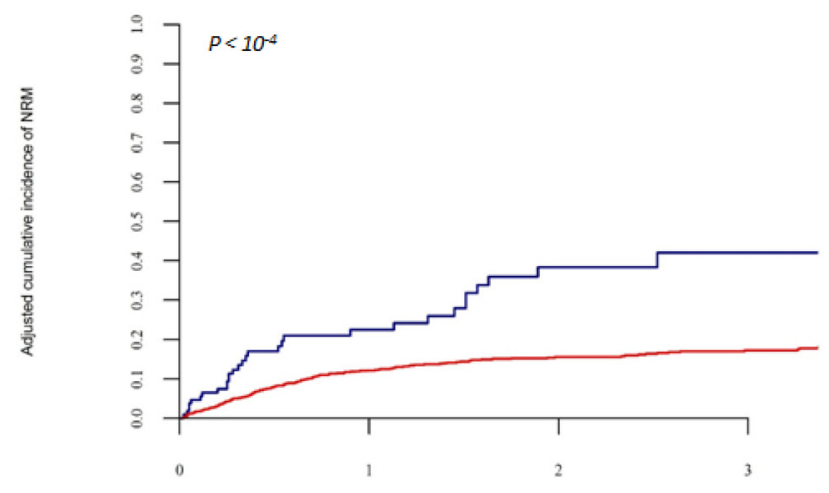

Time from transplant (years)

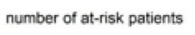

- TBF $\quad 147$

- Buflu 1459
RI

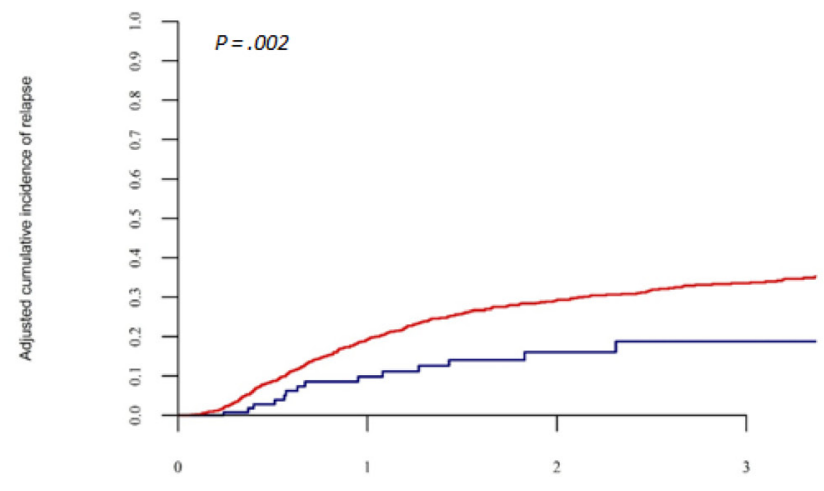

Time from transplant (years)

number of at-risk patients

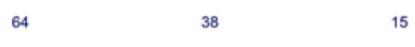

$682 \quad 425 \quad 293$

Figure 1: Non-relapse mortality and relapse incidence in patients receiving a myeloablative regimen. The cumulative incidence of non-relapse mortality and relapse by conditioning regimen (TBF-MAC vs BF-MAC).

LFS

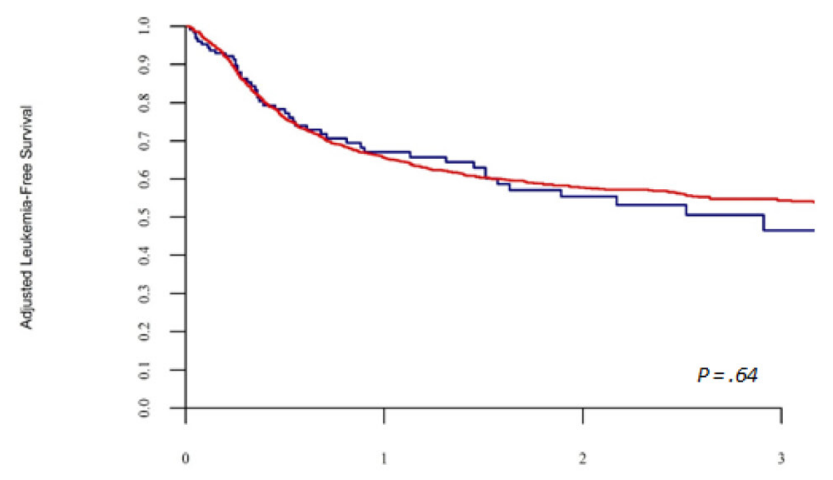

Time from transplant (years)

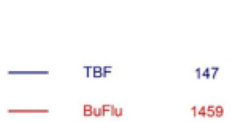

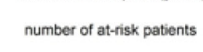

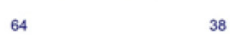

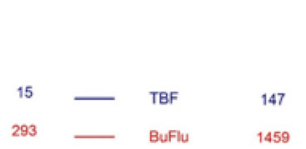
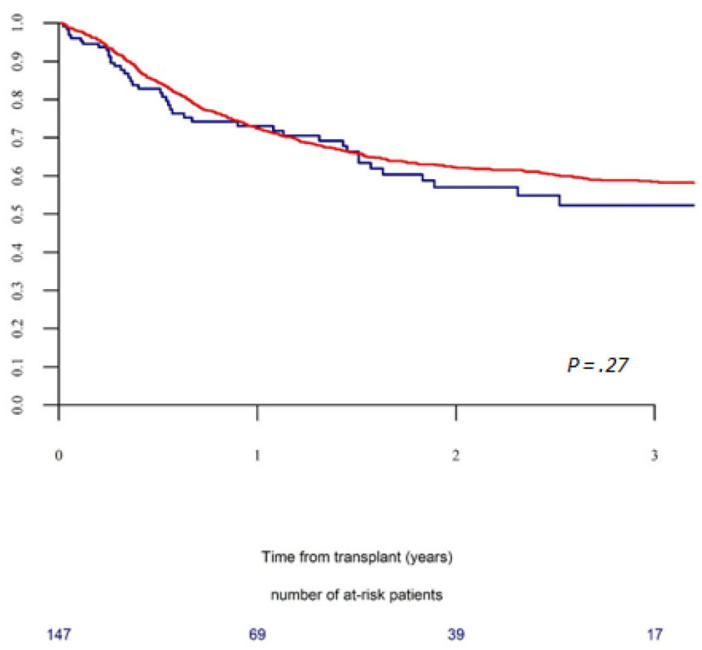

752

Figure 2: Leukemia free and overall survival in patients receiving a myeloablative regimen. The probabilities of leukemiafree and overall survival by conditioning regimen (TBF-MAC vs BF-MAC). 
Table 4: Multivariate analysis of transplantation outcome in TBF-MAC vs BF-MAC with selected busulfan dose

\begin{tabular}{|c|c|c|c|c|}
\hline & Outcome & HR & $95 \% \mathrm{CI}$ & $P$ \\
\hline \multirow[t]{8}{*}{ Relapse } & TBF vs BF & 0.45 & $0.25-0.83$ & 0.01 \\
\hline & Patient age per 10 years & 1.13 & $1.02-1.25$ & 0.02 \\
\hline & Secondary AML & 1.25 & $0.88-1.77$ & 0.2 \\
\hline & URD vs MSD & 1.11 & $0.81-1.52$ & 0.49 \\
\hline & Female donor to male recipient & 0.96 & $0.72-1.29$ & 0.8 \\
\hline & In-vivo TCD & 0.82 & $0.62-1.09$ & 0.19 \\
\hline & PBSC vs BM & 0.96 & $0.72-1.29$ & 0.81 \\
\hline & centre (frailty) & & & 0.69 \\
\hline \multirow[t]{8}{*}{ NRM } & TBF vs BF & 1.53 & $0.89-2.6$ & 0.12 \\
\hline & Patient age per 10 years & 1.57 & $1.35-1.82$ & $<10^{-5}$ \\
\hline & Secondary AML & 0.88 & $0.56-1.38$ & 0.58 \\
\hline & URD vs MSD & 1.61 & $1.08-2.38$ & 0.02 \\
\hline & Female donor to male recipient & 1.12 & $0.79-1.58$ & 0.53 \\
\hline & In-vivo TCD & 0.814 & $0.562-1.177$ & 0.27 \\
\hline & PBSC vs BM & 1.94 & $1.21-3.12$ & 0.006 \\
\hline & centre (frailty) & & & 0.004 \\
\hline \multirow[t]{8}{*}{ LFS } & TBF vs BF & 0.82 & $0.56-1.2$ & 0.31 \\
\hline & Patient age per 10 years & 1.26 & $1.16-1.38$ & $<10^{-5}$ \\
\hline & Secondary AML & 1.08 & $0.82-1.42$ & 0.59 \\
\hline & URD vs MSD & 1.27 & $0.99-1.61$ & 0.059 \\
\hline & Female donor to male recipient & 1.03 & $0.82-1.29$ & 0.79 \\
\hline & In-vivo TCD & 0.8 & $0.63-1.01$ & 0.05 \\
\hline & PBSC vs BM & 1.27 & $0.98-1.64$ & 0.07 \\
\hline & centre (frailty) & & & 0.22 \\
\hline \multirow[t]{8}{*}{ OS } & TBF vs BF & 0.95 & $0.63-1.44$ & 0.82 \\
\hline & Patient age per 10 years & 1.32 & $1.19-1.45$ & $<10^{-5}$ \\
\hline & Secondary AML & 0.99 & $0.73-1.33$ & 0.92 \\
\hline & URD vs MSD & 1.21 & $0.92-1.58$ & 0.17 \\
\hline & Female donor to male recipient & 1.02 & $0.81-1.3$ & 0.85 \\
\hline & In-vivo TCD & 0.82 & $0.64-1.06$ & 0.13 \\
\hline & PBSC vs BM & 1.37 & $1.03-1.82$ & 0.03 \\
\hline & centre (frailty) & & & 0.14 \\
\hline \multirow[t]{8}{*}{ GRFS } & TBF vs BF & 0.78 & $0.54-1.126$ & 0.18 \\
\hline & Patient age per 10 years & 1.21 & $1.118-1.319$ & $<10^{-5}$ \\
\hline & Secondary AML & 1.04 & $0.807-1.344$ & 0.76 \\
\hline & URD vs MSD & 1.21 & $0.963-1.52$ & 0.10 \\
\hline & Female donor to male recipient & 1.25 & $1.027-1.517$ & 0.76 \\
\hline & In-vivo TCD & 0.75 & $0.595-0.933$ & 0.01 \\
\hline & PBSC vs BM & 1.29 & $1.007-1.663$ & 0.04 \\
\hline & centre (frailty) & & & 0.02 \\
\hline
\end{tabular}

17] we conducted a subgroup analysis of TBF-MAC patients who received busulfan $9.6 \mathrm{mg} / \mathrm{kg}$, which were compared to BF-MAC patients receiving busulfan 12.8 $\mathrm{mg} / \mathrm{kg}$ (Table 4). With the reduced dose of busulfan (9.6 $\mathrm{mg} / \mathrm{kg}$ ) the NRM risk following TBF-MAC resulted still higher however not significantly different as compared to BF-MAC (HR 1.53, $p=0.12$ ). Notably, despite the reduced busulfan dosage, relapse risk following TBFMAC remained significantly lower as compared to BFMAC (HR 0.45, $p=0.01$ ) (Figure 3). Finally, no impact 
of the kind of conditioning regimen could be observed on LFS, OS or GRFS (TBF-MAC vs BF-MAC: LFS: HR 0.82, $p=0.31$; OS: HR 0.95, $p=0.82$; GRFS: HR $0.78, p=0.18)$. Results of multivariate analysis in this subgroup are presented in Table 4.

\section{Propensity score matched-pairs analysis}

Primary and secondary endpoints were further challenged in a propensity score matched-pairs analysis, conducted on 138 TBF-MAC matched with 262 BF-MAC patients, which confirmed the results obtained in the global population (Supplementary Tables 1 and 2, appendix).

\section{Reduced-intensity conditioning: TBF-RIC versus BF-RIC}

One thousand three hundred and four patients received a RIC regimen; among them, 65 patients conditioned with TBF-RIC were compared to 1239 patients who received BF-RIC. Characteristics of patients are summarized in Table 1.

Engraftment rate was $96 \%$ following TBF-RIC and $99 \%$ after BF-RIC, respectively $(p=0.063)$. The median time to neutrophil engraftment was 16 (8-29) days and 18 (8-97) days for TBF-RIC and BF-RIC, respectively $(p=0.01)$. The incidence of grade II-IV and III-IV aGVHD was similar between the groups, being $22 \pm 9 \%$

\section{NRM}
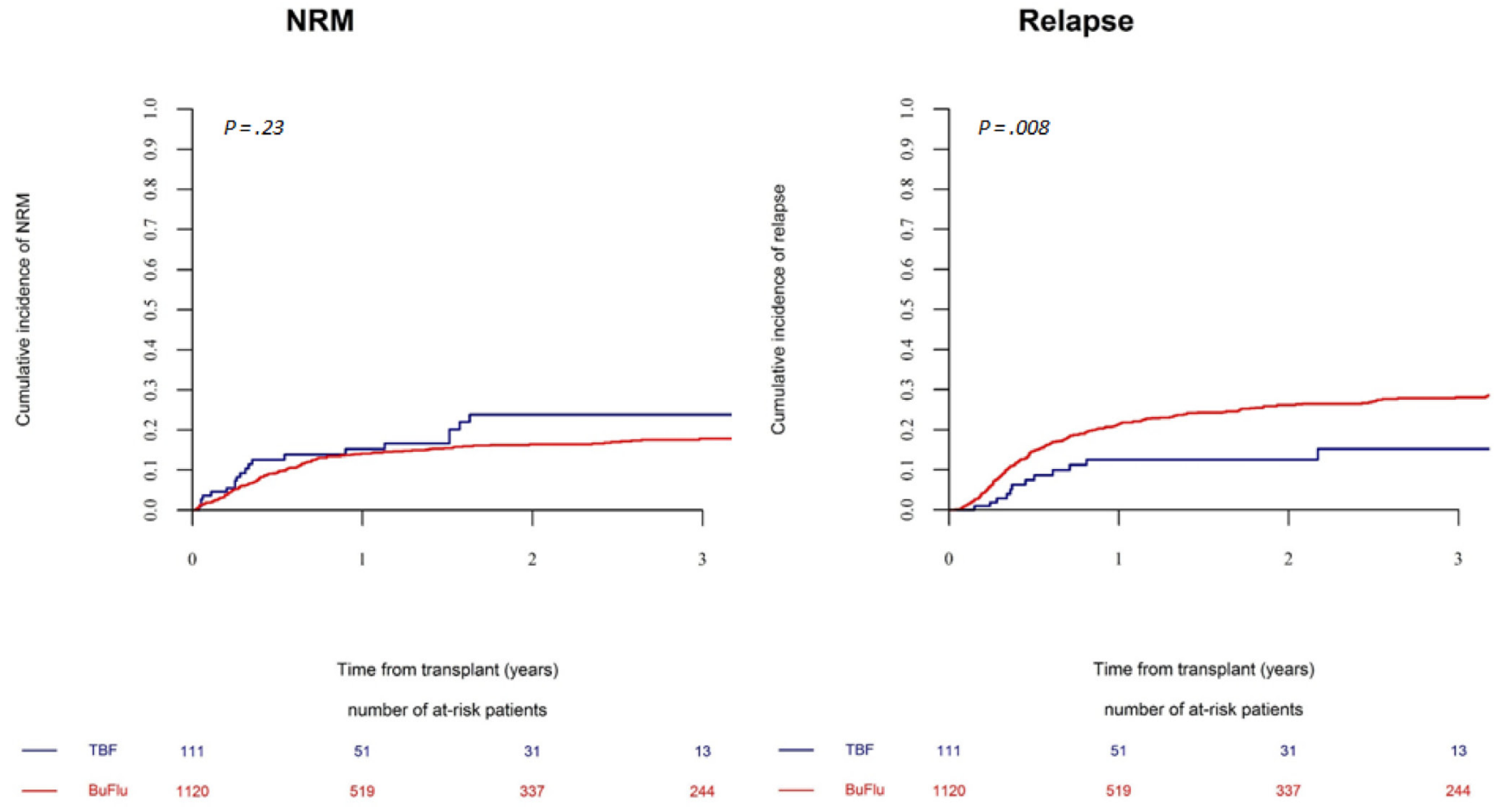

and $6 \pm 4 \%$ in the TBF-RIC and $22 \pm 3 \%$ and $8 \pm 2 \%$ in the BF-RIC group ( $p=0.79$ and $p=0.59)$, respectively.

Leukemia-free survival and overall survival did not differ between TBF-RIC and BF-RIC, being $47 \pm 18 \%$ vs $52 \pm 3 \%(p=0.43)$ and $60 \pm 17 \%$ vs $57 \pm 3 \%(p=0.81)$, respectively (Figure 4).

Multivariate analysis confirmed those results (Table 3). The 2-year NRM and relapse rates were not statistically different between the two study groups, being $15 \pm 8 \%$ vs $18 \pm 2 \%(p=0.62)$ and $39 \pm 18 \%$ vs $30 \pm 3 \%$ $(p=0.62)$, respectively. The 2 -year cumulative incidence of cGVHD was similar between the groups, and so was the composite endpoint GRFS.

The use of ATG was independently associated with significantly lower probability of severe cGVHD and better GRFS.

Similarly to the MAC cohort, endpoints were further tested by a propensity score matched-pairs analysis, conducted on 61 TBF-RIC vs 118 BF-RIC patients, which confirmed these results (Supplementary Tables 1 and 2, appendix).

\section{DISCUSSION}

Despite great efforts in optimizing preparatory regimens and transplant procedures, leukemia relapse remains today the major cause of transplant failure. We hypothesized that the thiotepa-busulfan-fludarabine

Figure 3: Non-relapse mortality and relapse incidence in patients receiving a myeloablative regimen with selected busulfan doses. The cumulative incidence of relapse and non-relapse mortality by conditioning regimen (TBF with busulfan $9.6 \mathrm{mg} / \mathrm{kg}$ vs BF with busulfan $12.8 \mathrm{mg} / \mathrm{kg}$ ). 
protocol could ensure a strong leukemia control, reducing relapse and thus leading to improved outcome as compared to busulfan-fludarabine.

In fact, the TBF-MAC conditioning was associated with inferior relapse as compared to BF-MAC; however, the increased non-relapse mortality offset this advantage and no difference could be observed in survival between the two regimens. Notably, the 2-year relapse incidence was as low as 14\% following the TBF-MAC; in multivariate analysis, relapse risk was cut by half using the TBF-MAC as compared to the BF-MAC protocol. These results compare favourably with myeloablative regimens currently employed; for instance, 2-year relapse rate following the classical $\mathrm{CY} / \mathrm{TBI}$ regimen stands at about 20\% when transplant is given in remission [25]. In fact, leukemia recurrence following transplant remains an unresolved issue, and the recent tendency to mitigate conditioning regimen intensity raises significant concerns about further increasing relapse risk, as confirmed by recent evidence [26]. In their randomized $\mathrm{BF}$ vs $\mathrm{BuCy}$ study, Lee and colleagues [8] reported a 2-year relapsefree survival of $55 \%$ following $\mathrm{BF}$, which was significantly inferior as compared to BuCy; Rambaldi et al. [10] observed a cumulative incidence of relapse following BF of about $30 \%$ at 2 years which however, in contrast to the previously cited study, was not statistically different as compared to $\mathrm{BuCy}$.

The addition of thiotepa to busulfan and fludarabine appears to provide a significantly stronger anti leukemic effect; in fact, our results concur with recent observations reporting encouraging low relapse rates when TBF was employed in cord blood (Sanz et al., [17] 5-year RI: 18\%) and haplo-SCT (Bacigalupo et al., [19] cumulative incidence of relapse related death: $11 \%$ for patients in CR1). In the

LFS

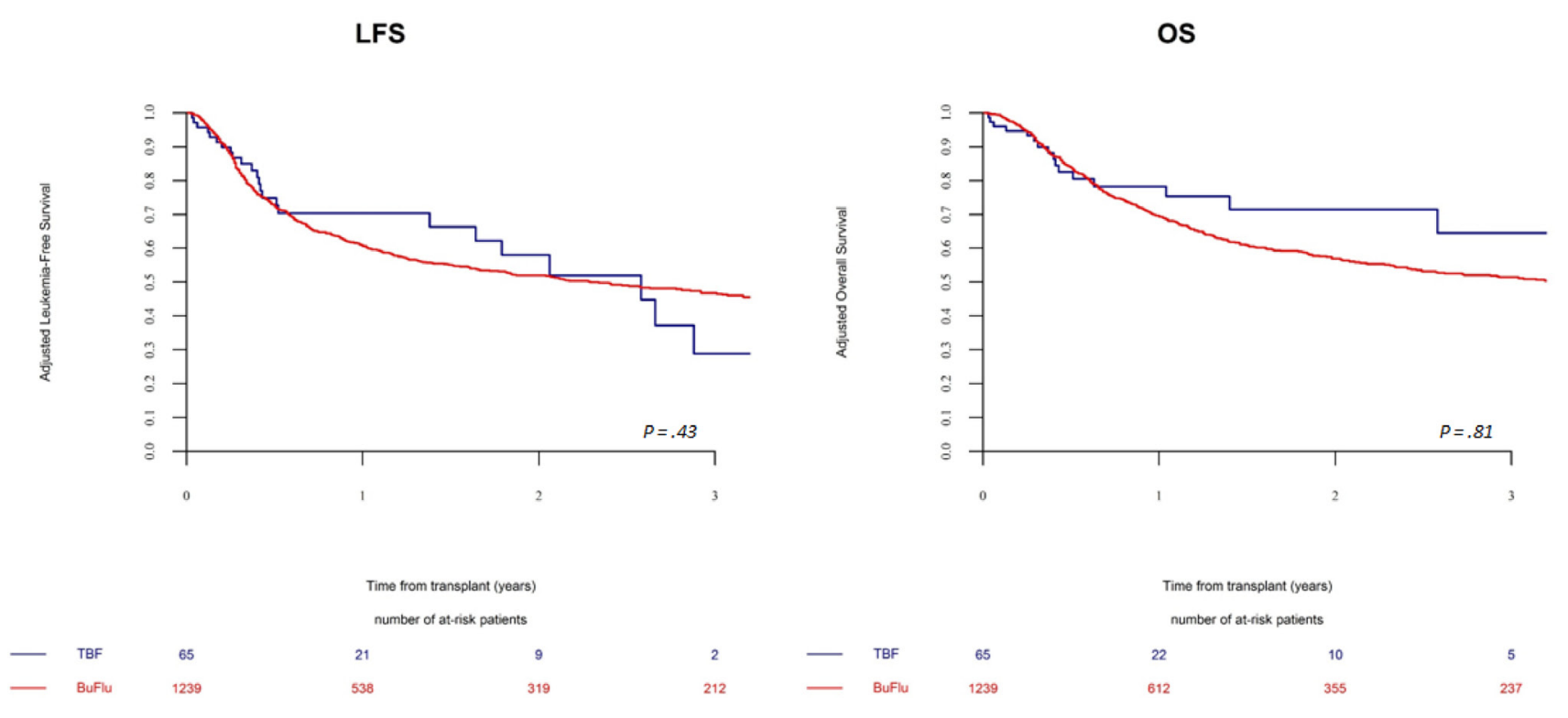

study by Di Bartolomeo et al., [21] the use of TBF-MAC was the only factor predicting lower relapse risk in multivariate analysis. It might be speculated that the combined efficacy of two alkylating agents mediating powerful anti-leukemic activity could be responsible of such effective disease control. In addition, thiotepa is well known to have a radiomimetic action and to penetrate sanctuary sites [27].

Nevertheless, the low relapse rate following TBF observed in our study should be interpreted with caution as it could, at least in part, be related to the high NRM observed in the same cohort of patients. Indeed, 2-year NRM following TBF-MAC was $27 \%$ in the overall population, which resulted significantly higher as compared to the BF conditioning. Higher NRM following a strong myeloablative protocol (TBF) as compared to a reduced-toxicity regimen $(\mathrm{BF})$ does not represent a surprising finding per se; further, this result is in accordance with the previously cited studies in which TBFMAC was employed in haploidentical (Arcese et al. [22] cumulative incidence of NRM: 32\%) and cord blood transplant (Sanz et al., [17] 5-year NRM: 44\%). However, the significant NRM rate reported in our study warrants a deeper reflection. When examining factors associated with NRM, busulfan dose (12.8 vs $9.6 \mathrm{mg} / \mathrm{kg}$ ) stood out as strongly predicting high NRM risk, while it did not demonstrate any impact on relapse incidence. In view of this observation, and considering the published standard regimen schedules, $[10,17]$ we performed a subgroup analysis comparing TBF-MAC with busulfan $9.6 \mathrm{mg} / \mathrm{kg}$ and BF-MAC with busulfan $12.8 \mathrm{mg} / \mathrm{kg}$. With the reduced dose of busulfan, NRM risk after TBF substantially decreased to a level not statistically different as compared to BF; however a trend towards higher NRM for TBF was retained. Importantly, the lower dose of busulfan did

Figure 4: Leukemia free and overall survival in patients receiving a reduced intensity regimen. The probabilities of leukemia-free and overall survival by conditioning regimen (TBF-RIC vs BF-RIC). 
not impair the anti-leukemic activity of TBF, as relapse remained significantly inferior as compared to BF. Based on these data, the combination of two alkylators at full myeloablative doses appears excessively toxic, with no apparent benefit in terms of disease control. Nevertheless in this subgroup analysis, similarly to what was found in the global population, the strong advantage of TBF-MAC in terms of reduced relapse was negated by the high (although slightly reduced) NRM. Indeed, despite TBF hazard ratios for LFS, OS and GRFS resulted constantly below 1, no statistical difference in survival could be observed between the two regimens.

When conducting exploratory analyses according to age, donor type and leukemia risk, we could not find any significant difference between TBF-MAC and BF-MAC in terms of survival in any of the subgroups examined.

The main causes of death were infection and GVHD. Lethal infectious complications accounted for one out of four deaths in the TBF group. Our results are in accordance with previous publications reporting high rate of infection following TBF regimen [22]. Thiotepa holds a heavy myeloablative effect combined with a powerful immunosuppressive potential which may have contributed to this. The acknowledgment of such risks following TBF-MAC could help to better select patients suitable for this regimen, and to implement an optimal anti-infectious monitoring, prophylaxis and treatment which may improve outcome.

Notably, despite the higher intensity, TBF-MAC resulted in similar incidence of grade II-IV aGVHD as compared to BF-MAC. The strong immunosuppressive activity of thiotepa in association with fludarabine may have played a role in this. Finally, incidence of severe cGVHD was similar between the two conditioning regimens. Importantly, the inclusion of ATG in both protocols was independently associated with significantly lower probability of severe cGVHD both in patients receiving MAC and RIC, and with better GRFS.

In a separate analysis, we compared reduced-intensity versions of $\mathrm{TBF}$ and $\mathrm{BF}$, reporting similar outcome. Interestingly, NRM rate of TBF-RIC was similar to BFRIC, despite the combination of two alkylators in a cohort of patients with a median age of 60 years. We did not observe a difference in relapse incidence between the two study cohorts; however, due to the limited number of patients receiving TBF-RIC no definite conclusion can be made.

It is important to recognize the limitations of the present study. First, the retrospective design did not allow to study the reason for patient allocation to a specific regimen. Secondly, the two study arms were unbalanced as to the number of patients included. Finally, some of the patient's characteristics varied among the two groups. Nonetheless, the present analysis represents the largest study reporting outcome of TBF conditioning in MSD or URD-SCT for AML; further, we addressed the inherent limitations of a registry-based study performing a confirmatory PS matched-pair analysis, which thoroughly upheld all the results we observed in the overall population.

\section{CONCLUSIONS}

In conclusion, our results suggest that TBF-MAC provides significantly lower relapse, which is counterbalanced by increased NRM as compared to BF-MAC, thus resulting in similar survival. The combination of thiotepa with high dose busulfan $(12.8 \mathrm{mg} / \mathrm{kg})$ appears excessively toxic, while TBF with lower dose of busulfan $(9.6 \mathrm{mg} / \mathrm{kg})$ seems to retain strong anti-leukemic effect in combination with acceptable NRM, however with no statistically significant survival advantage over BF. In elderly patients, TBF-RIC appears a promising reduced-intensity regimen, as similar outcome was reported as compared to BF-RIC. This registry data should be validated by a well-designed randomized trial comparing outcome of TBF with other busulfan-based regimens, both in the MAC and RIC setting. Specifically, designed studies are necessary to identify which patient category could benefit the most from the strong anti-leukemic potential of TBF protocol, without an excess of NRM. Young, fit patients at very high risk of relapse might candidate for TBF regimen, providing a careful patient selection and an optimal supportive care in order to minimize transplant toxicity.

\section{METHODS}

\section{Study design and data collection}

This is a registry based retrospective study. Data were provided and the study design was approved by the acute leukemia working party (ALWP) of the EBMT group registry, in accordance with the EBMT guidelines for retrospective studies. EBMT is a voluntary working group of more than 500 transplant centers which are required to report all consecutive stem cell transplantations and follow-up once a year. Audits are routinely performed to determine the accuracy of the data. Since 1990, patients have been able to provide informed consent that authorizes the use of their transplant information for research purposes. The ALWP of the EBMT granted ethical approval for this study. We included in the analysis patients with AML older than 18 at diagnosis, who had received either TBF or BF as conditioning regimen for MSD or URD SCT in CR1 as first transplant between January 2007 and June 2015, reported to the EBMT. All unrelated donors were HLA-matched (10/10) or mismatched at one HLA locus (9/10). Patients who received conditioning regimens including oral busulfan, T-depleted grafts, or transplant from $<9 / 10$ mismatched unrelated donor were excluded. Myeloablative conditioning regimen (MAC) was defined as ivBusulfan dose $\geq 9.6 \mathrm{mg} / \mathrm{kg}$ (TBF-MAC and BF-MAC), while reduced-intensity conditioning (RIC) was defined as iv Busulfan dose of $6.4 \mathrm{mg} / \mathrm{kg}$ 
(TBF-RIC and BF-RIC) in accordance with the EBMT definitions [28].

\section{End-point definitions and statistical analysis}

Primary end-points were overall survival (OS) and leukemia-free survival (LFS). Secondary end-points were relapse incidence (RI), non-relapse mortality (NRM), graft-versus-host free, relapse-free survival (GRFS), engraftment, incidence and severity of acute (aGVHD) and chronic graft-versus-host disease (cGVHD). The severity of acute GVHD was graded on a I-IV scale, while cGVHD was scored as mild, moderate or severe in accordance to EBMT standards [28]. LFS was defined as the interval from transplant to either relapse or death. OS was defined as the time between the date of transplant and the date of death. GRFS was defined as alive with no previous grade III-IV aGvHD, no severe chronic GvHD and no relapse [29]. Probabilities of OS, LFS and GRFS were estimated using Kaplan-Meier curves. Cumulative incidence functions were used to estimate relapse incidence (RI) and non-relapse mortality (NRM) in a competing risks setting. In order to study acute and chronic GVHD, we considered death and relapse as competing events. The main patient characteristics were compared using Mann-Whitney test for quantitative variables, chi-square test or Fisher exact test for categorical variables. Univariate analyses were performed using the log rank test for OS, LFS and GRFS, the Gray test for cumulative incidences. Multivariate analyses were performed using the Cox proportionalhazard model. Factors differing between two groups in terms of distribution and factors significantly associated with the outcome were included in the multivariate analysis. In order to test for a centre effect, we introduced a random effect of frailty for each centre into the model [30]. Finally, a propensity score matched pairs analysis was conducted to corroborate the results obtained in the global population; detailed statistics and results are presented in the appendix. All tests were two-sided and $P$ values $<0.05$ were considered as indicating a statistically significant association. Analyses were performed using the R statistical software version 3.2.3 (available online at http://www.R-project.org), and propensity score analysis was performed using the 'MatchIt' [31].

\section{Abbreviations}

aGVHD: acute graft-versus-host disease; BF: busulfan-fludarabine; BM: Bone marrow; Bu: busulfan; cGVHD: chronic graft-versus-host disease; GRFS: graft-versus-host-free, relapse-free survival; MAC: myeloablative conditioning; LFS: leukemia-free survival; MSD: matched sibling donor; NRM: non-relapse mortality OS: overall survival; URD: unrelated donor; PBSCs: peripheral blood stem cells; RIC: reduced-intensity conditioning; TBF: thiotepa-busulfan-fludarabine; TCD: T-cell depletion.

\section{Author contributions}

F.S. and A.N. designed the study, the synopsis of which was approved by the acute leukemia working party of the EBMT; M.L. performed all the statistical analysis; F.S. wrote the first draft of the manuscript; A.N. and B.S. reviewed the manuscript; all co-authors contributed data to the EBMT registry, read the manuscript and approved the final version.

\section{ACKNOWLEDGMENTS}

The authors would like to thank all EBMT centers for contributing patients to the study and data managers for their great work. A complete list of the EBMT members appears in the supplement.

\section{CONFLICTS OF INTEREST}

The authors declare no conflicts of interest.

\section{FUNDING SUPPORT}

No specific funding to be disclosed.

\section{Editorial note}

This paper has been accepted based in part on peerreview conducted by another journal and the authors' response and revisions as well as expedited peer-review in Oncotarget.

\section{REFERENCES}

1. Shimoni A, Nagler A. Optimizing the conditioning regimen for allogeneic stem-cell transplantation in acute myeloid leukemia; dose intensity is still in need. Best Pract Res Clin Haematol. 2011; 24:369-79.

2. Russell JA, Tran HT, Quinlan D, Chaudhry A, Duggan P, Brown C, Stewart D, Ruether JD, Morris D, Glick S, Gyonyor E, Andersson BS. Once-daily intravenous busulfan given with fludarabine as conditioning for allogeneic stem cell transplantation: study of pharmacokinetics and early clinical outcomes. Biol Blood Marrow Transplant. 2002; 8:468-476.

3. Bornhauser M, Storer B, Slattery JT, Appelbaum FR, Deeg HJ, Hansen J, Martin PJ, McDonald GB, Nichols WG, Radich J, Woolfrey A, Jenke A, Schleyer E, et al. Conditioning with fludarabine and targeted busulfan for transplantation of allogeneic hematopoietic stem cells. Blood. 2003; 102:820-6. 
4. Bredeson $\mathrm{CN}$, Zhang MJ, Agovi MA, Bacigalupo A, Bahlis NJ, Ballen K, Brown C, Chaudhry MA, Horowitz MM, Kurian S, Quinlan D, Muehlenbien CE, Russell JA, et al. Outcomes following HSCT using fludarabine, busulfan, and thymoglobulin: a matched comparison to allogeneic transplants conditioned with busulfan and cyclophosphamide. Biol Blood Marrow Transplant. 2008; 14:993-1003.

5. Chae YS, Sohn SK, Kim JG, Cho YY, Moon JH, Shin HJ, Chung JS, Cho GJ, Yang DH, Lee JJ, Kim YK, Kim HJ. New myeloablative conditioning regimen with fludarabine and busulfan for allogeneic stem cell transplantation: comparison with BuCy2. Bone Marrow Transplant. 2007; 40:541-47.

6. Andersson BS, de Lima M, Thall PF, Wang X, Couriel D, Korbling M, Roberson S, Giralt S, Pierre B, Russell JA, Shpall EJ, Jones RB, Champlin RE. Once daily i.v. busulfan and fludarabine (i.v. Bu-Flu) compares favorably with i.v. busulfan and cyclophosphamide (i.v. BuCy2) as pretransplant conditioning therapy in AML/MDS. Biol Blood Marrow Transplant. 2008; 14:672-84.

7. Pasquini MC, Le-Rademacher J, Zhu X, Artz A, DiPersio J, Fernandez HF, Mineishi S, Kamishohara M, Mehta J, Nakamura Y, Ratanatharathorn V, Sobecks R, Burkart J, et al. Intravenous Busulfan-Based Myeloablative Conditioning Regimens Prior to Hematopoietic Cell Transplantation for Hematologic Malignancies. Biol Blood Marrow Transplant. 2016; 22:1424-1430.

8. Lee JH, Joo YD, Kim H, Ryoo HM, Kim MK, Lee GW, Lee JH, Lee WS, Park JH, Bae SH, Hyun MS, Kim DY, Kim SD, et al. Randomized trial of myeloablative conditioning regimens: busulfan plus cyclophosphamide versus busulfan plus fludarabine. J Clin Oncol. 2013; 31:701-9.

9. Liu H, Zhai X, Song Z, Sun J, Xiao Y, Nie D, Zhang Y, Huang F, Zhou H, Fan Z, Tu S, Li Y, Guo X, et al. Busulfan plus fludarabine as a myeloablative conditioning regimen compared with busulfan plus cyclophosphamide for acute myeloid leukemia in first complete remission undergoing allogeneic hematopoietic stem cell transplantation: a prospective and multicenter study. J Hematol Oncol. 2013; 6:15.

10. Rambaldi A, Grassi A, Masciulli A, Boschini C, Micò MC, Busca A, Bruno B, Cavattoni I, Santarone S, Raimondi R, Montanari M, Milone G, Chiusolo P, et al. Busulfan plus cyclophosphamide versus busulfan plus fludarabine as a preparative regimen for allogeneic haemopoietic stem-cell transplantation in patients with acute myeloid leukaemia: an open-label, multicentre, randomised, phase 3 trial. Lancet Oncol. 2015; 16:1525-36.

11. Liu DH, Xu LP, Zhang XH, Wang Y, Yan CH, Wang JZ, Wang FR, Sun YQ, Ji Y, Zhang YY, Liu KY, Huang XJ. Substitution of cyclophosphamide in the modified BuCy regimen with fludarabine is associated with increased incidence of severe pneumonia: A prospective, randomized study. Int J Hematol. 2013; 98:708-715.

12. Ben-Barouch S, Cohen O, Vidal L, Avivi I, Ram R. Busulfan fludarabine vs busulfan cyclophosphamide as a preparative regimen before allogeneic hematopoietic cell transplantation: systematic review and meta-analysis. Bone Marrow Transplant. 2016; 51:232-40.

13. Aversa F, Tabilio A, Velardi A, Cunningham I, Terenzi A, Falzetti F, Ruggeri L, Barbabietola G, Aristei C, Latini P, Reisner Y, Martelli MF, Felicini R, et al. Treatment of highrisk acute leukemia with T-cell-depleted stem cells from related donors with one fully mismatched HLA haplotype. N Engl J Med. 1998; 339:1186-93.

14. Rosales F, Naparstek E, Varadi G, Or R, Slavin S, Nagler A. The role of thiotepa in allogeneic stem cell transplantation in patients with leukemia. Leuk Res. 1999; 23:947-52.

15. Eder S, Labopin M, Finke J, Bunjes D, Olivieri A, Santarone S, Rambaldi A, Kanz L, Messina G, Mohty M, Nagler A. Safety and efficacy of thiotepa-based conditioning for allogeneic transplantation in AML: a survey from the ALWP of the EBMT. Bone Marrow Transplant. 2017; 52:238-44.

16. Sanz J, Sanz MA, Saavedra S, Lorenzo I, Montesinos P, Senent L, Planelles D, Larrea L, Martín G, Palau J, Jarque I, Martínez J, de la Rubia J, et al. Cord blood transplantation from unrelated donors in adults with high-risk acute myeloid leukemia. Biol Blood Marrow Transplant. 2010; 16:86-94.

17. Sanz J, Boluda JC, Martín C, González M, Ferrá C, Serrano D, de Heredia CD, Barrenetxea C, Martinez AM, Solano C, Sanz MA, Sanz GF; Grupo Español de Trasplante Hematopoyético y Terapia Celular (GETH). Singleunit umbilical cord blood transplantation from unrelated donors in patients with hematological malignancy using busulfan, thiotepa, fludarabine and ATG as myeloablative conditioning regimen. Bone Marrow Transplant. 2012; 47:1287-93.

18. Raiola AM, Dominietto A, Ghiso A, Di Grazia C, Lamparelli T, Gualandi F, Bregante S, Van Lint MT, Geroldi S, Luchetti S, Ballerini F, Miglino M, Varaldo R, et al. Unmanipulated haploidentical bone marrow transplantation and posttransplantation cyclophosphamide for hematologic malignancies after myeloablative conditioning. Biol Blood Marrow Transplant. 2013; 19:117-22.

19. Bacigalupo A, Dominietto A, Ghiso A, Di Grazia C, Lamparelli T, Gualandi F, Bregante S, Van Lint MT, Geroldi S, Luchetti S, Grasso R, Pozzi S, Colombo N, et al. Unmanipulated haploidentical bone marrow transplantation and post-transplant cyclophosphamide for hematologic malignancies following a myeloablative conditioning: an update. Bone Marrow Transplant. 2015; 50:S37-9.

20. Bregante S, Dominietto A, Ghiso A, Raiola AM, Gualandi F, Varaldo R, Di Grazia C, Lamparelli T, Luchetti S, Geroldi S, Casarino L, Pozzi S, Tedone E, et al. Improved Outcome of Alternative Donor Transplantations in Patients with Myelofibrosis: From Unrelated to Haploidentical Family Donors. Biol Blood Marrow Transplant. 2016; 22:324-9.

21. Di Bartolomeo P, Santarone S, De Angelis G, Picardi A, Cudillo L, Cerretti R, Adorno G, Angelini S, Andreani M, 
De Felice L, Rapanotti MC, Sarmati L, Bavaro P, et al. Haploidentical, unmanipulated, G-CSF-primed bone marrow transplantation for patients with high-risk hematologic malignancies. Blood. 2013; 121:849-57.

22. Arcese W, Picardi A, Santarone S, De Angelis G, Cerretti R, Cudillo L, Pennese E, Bavaro P, Olioso P, Dentamaro T, Cupelli L, Chierichini A, Ferrari A, et al. Haploidentical, G-CSF-primed, unmanipulated bone marrow transplantation for patients with high-risk hematological malignancies: an update. Bone Marrow Transplant. 2015; 50:S24-30.

23. Cerretti R, Picardi A, Cudillo L, De Angelis G, Mariotti B, Mengarelli A, Marchesi F, Mangione I, Testi M, De Fabritiis P, Dentamaro T, Tirindelli MC, Saltarelli F, et al. Unique Chemotherapy Based Reduced Intensity Conditioning Regimen for Patients Transplanted from HLA Identical Siblings, Haploidentical or Volunteer Unrelated Donors: a Prospective Study from the Rome Transplant Network. Bone Marrow Transplant. 2015; 50.

24. Picardi A, Cudillo L, Mangione I, De Angelis G, Mariotti B, Di Piazza F, Cantonetti M, Ceresoli E, Rapanotti MC, de Fabritiis P, Dentamaro T, Cristina Tirindelli M, Annibali O, et al. The Impact of HLA Matching on the Clinical Outcome of Hematopoietic Stem Cell Transplantation from Volunteer Unrelated Donors According to the Uniform, Prospective Transplant Program of the Rome Transplant Network. Bone Marrow Transplant. 2015; 50.

25. Nagler A, Rocha V, Labopin M, Unal A, Ben Othman T, Campos A, Volin L, Poire X, Aljurf M, Masszi T, Socie G, Sengelov H, Michallet M, et al. Allogeneic hematopoietic stem-cell transplantation for acute myeloid leukemia in remission: comparison of intravenous busulfan plus cyclophosphamide $(\mathrm{Cy})$ versus total-body irradiation plus $\mathrm{Cy}$ as conditioning regimen-a report from the acute leukemia working party of the European group for blood and marrow transplantation. J Clin Oncol. 2013; 31:3549-56.

26. Scott BL, Pasquini MC, Logan BR, Wu J, Devine SM, Porter DL, Maziarz RT, Warlick ED, Fernandez HF, Alyea EP, Hamadani M, Bashey A, Giralt S, et al. Myeloablative Versus Reduced-Intensity Hematopoietic Cell Transplantation for Acute Myeloid Leukemia and Myelodysplastic Syndromes. J Clin Oncol. 2017; 35:1154-1161

27. Cheng T, Forsyth P, Chaudhry A, Morris D, Glück S, Russell JA, Stewart DA. High-dose thiotepa, busulfan, cyclophosphamide and ASCT without whole-brain radiotherapy for poor prognosis primary CNS lymphoma. Bone Marrow Transplant. 2003; 31:679-85.

28. Apperley J, Carreras E, Gluckman E, Masszi T, editors. The EBMT Handbook. 2012: 6th ed.

29. Ruggeri A, Labopin M, Ciceri F, Mohty M, Nagler A. Definition of GvHD-free, relapse-free survival for registrybased studies: an ALWP-EBMT analysis on patients with AML in remission. Bone Marrow Transplant. 2016; 51:610-1.

30. Andersen PK, Klein JP, Zhang MJ. Testing for centre effects in multi-centre survival studies: a Monte Carlo comparison of fixed and random effects tests. Stat Med. 1999; 18:1489-500.

31. Package 'MatchIt'. 2015 (accessed: 18 May 2015). https:// cran.r-project.org/web/packages/MatchIt/index.html. 